\title{
Issues of Variance of Extreme Values in a Heterogeneous Teletraffic Environment
}

\author{
D.E. Bassey ${ }^{1}$, R. C. Okoro ${ }^{2}$, B. E. Okon ${ }^{3}$ \\ ${ }^{1,2}$ Electronics and Computer Technology Unit, Department of Physics, University of Calabar, Cross River State, Nigeria
}

\begin{abstract}
The reliability of various teletraffic data-analytical devices in communication systems is relevant to all stakeholders. Among other key external variables, the performance of the network evaluated under a variety of traffic scenarios is the focus of the study: data / video traffic generated by subscribers, who ordinarily subscribed voice bandwidth services. This study, therefore views teletraffic data as complete random and in-deterministic variables, and examines its continuous dependence on configuration of fixed engineering values by equipment innovators/operators. The study was carried out by examining if core equipment techniques for processing traffic data on real-time basis vary in accordance with the bandwidth required to deliver the service (voice, data and video). Four Line Trunk Groups (LTG01, LTG02, LTG03 and LTG04) and 16 Digital Line Units (DLUs) were used for the study. Close examination of the activated DLUs configured to carry voice bandwidth generated daily traffic values of 0.1Erlang to 0.2Erlang, while others generated traffic rates of 0.01Erlang to $0.05 E$ Elang per line; during the non-busy-hour period. This traffic rates, though subscribed for voice services, were shared among voice, data and video. The study concludes that the time consistent busy hour is not truly consistent in the present heterogeneous traffic environment. New concepts were examined and recommendations made for review.
\end{abstract}

Keywords: Average Daily Peak Hour, Busy Hour, Digital Line Unit, Line Trunk Group, Peak Values, Time Consistent Busy Hour

\section{Introduction}

A switching unit or group of switching modules are usually determined by the rate at which traffic arrive and the length of time for which they are held. These two key qualities account for the term "traffic (or teletraffic)" in telecommunications engineering.

Since traffic arrive at random and they last for a random length of time, mean values are usually used in practice. Inter-arrival and holding time are determined by taking measurements over a long period and monitoring the pattern over traffic parameters. This approach, though lengthy and accurate within the place of measurements, forms the foundation of the teletraffic theorists' model.

The probability that a call/data arrives in an interval $t$, is not dependent on the time that has elapsed since the last call/data, or the holding time. The probability that a call releases in a given interval, $t$ is not related to the time for which the call has been in progress. The mean inter-arrival time is the inverse of the mean calling intensity, and therefore depends on the level of offered traffic. Usually, teletraffic theorists and administrators assume values ranging from 20 sec. to $180 \mathrm{sec}$. These values are dependent on time consistent voice calls [2].

In line with this theory, CCITT/ITU-T defined a representative hour called the Busy Hour [1]. Traffic density of this hour is used as the basis for network planning/ dimensioning. Traffic density is measured per quarter of an hour during a period of up to 15 working days. The representative hour, consisting of four consecutive quarters is chosen as the busiest hour. Two methods are usually used with respect to traffic measurements:

a. Time Consistent Busy Hour (TCBH), and

b. Average Daily Peak Hour (ADPH).
In $\mathrm{TCBH}$, the measurements per quarter are averaged. The Busy Hour that is determined remains constant in time, while traffic calculated is the average traffic value of the busy hour. TCBH is the most commonly used method. The ADPH adapts the method where a peak hour is determined and the traffic is calculated using the average traffic values of the daily peak hours. ADPH has no well determined busy hour as TCBH; rather, it has a busy period that can be one hour, or larger.

The reliability of these teletraffic-data measuring devices in communication systems is very important. These require the consideration of the effects of different external disturbances and a variety of traffic scenarios on the performance of the network. One of such scenarios is data/video traffic generated by subscribers who ordinarily subscribed low bandwidth services. Example: charting in face book, other social interacting web sites, and low bandwidth Internet users.

This study therefore views the present data dominated signal streams as completely random and in-deterministic. It further examines the rationale for the continuous dependence on fixed engineering service criteria by equipment innovators/operators. Should core equipment techniques for evaluating traffic data on real-time basis, vary in accordance with the present heterogeneous teletraffic environment? The objective is to ensure that modern traffic measurements, used for evaluating performance indicators may not need to activate restriction common with electro-mechanical switches, or digital switches with low bandwidth utilization; and real-time dependent. Fundamental concepts in traffic flow pattern between voice, data and video were evaluated. New concepts such as a review of the time-consisted busy hour as a catalyst to modify engineering and operational contents in communication networks analyses were examined and recommendations made for review. 


\section{International Journal of Science and Research (IJSR) \\ ISSN (Online): 2319-7064}

Index Copernicus Value (2013): 6.14 | Impact Factor (2014): 5.611

\section{Methodology}

The study was conducted using an EWSD switching system hardware, consisting of External Line Plant Terminal of 960 subscribers' interface Line-modules, 2000-digital communication pots, Operation and Maintenance Terminal, a Plug-in or Plug-out terminal with output registers for 32 Line Trunk Group Modules, located in the central equipment room, at the Calabar Export Processing Zone, CalabarNigeria. It serves both foreigners and indigenous workers. Using the real-time traffic hourly mode, time consistent hourly traffic data were obtained to validate the process. Another, un-automated traffic measurements were simultaneously activated with the aid of a Dial-Tone Delay Recorder. The device measured traffic by placing test calls to selected routes and measured service delays over 3 seconds. Digital Line Units (DLUs) selected for these tests were configured to carry traffic for two distinct subscribers separated by nationality and volume of traffic usage. Through this unit, a deliberate step-by-step test calls were made and a measure of the proportion of time that all the subscriber line modules were found busy in each Line Trunk Group noted. The resulting occupancy of all DLUs found busy is a direct measure of the probability of a delay $>0$. The peak mode configuration was activated throughout the process.

The main processor load measurement provides an overview of the load situation regarding selected DLUs or LTGs. The required load per DLU is recorded using the man-machine language command: Traffic measurement of DLU- 01 to DLU-10 (CR MPLDME DLU 01-10).

START TIME: Time of output of the first report for 60 mins. Interval: 0000 hrs to 2400 hrs.

The output data were recorded using the magnetic disk drive and the magnetic tape drive.

\section{Results and Discussion}

Subscriber observation command (ENTR DNOBS) generated data records for all incoming and outgoing traffic under observation. It provided information about how subscriber traffic was distributed over the various traffic routes and supplied values relating to traffic behavior. Post processing of data can be used to determine traffic variables for individual subscriber or group of subscribers.

Fig. 1. is a comparative data acquired under similar conditions using two different measuring devices: computerized system and the manually operated device. Measurements were carried out to determine the traffic busy hour of the switch. Fig. 2 to Fig. 11 are mean traffic values in Erlang recorded using the time frame of the two major traffic dips recorded in Fig. 1. , 0800 hours to 1500 hours and 1500 hours to 2400 hours. The readings were taken in three consecutive days, each, under a stretch of six weeks.

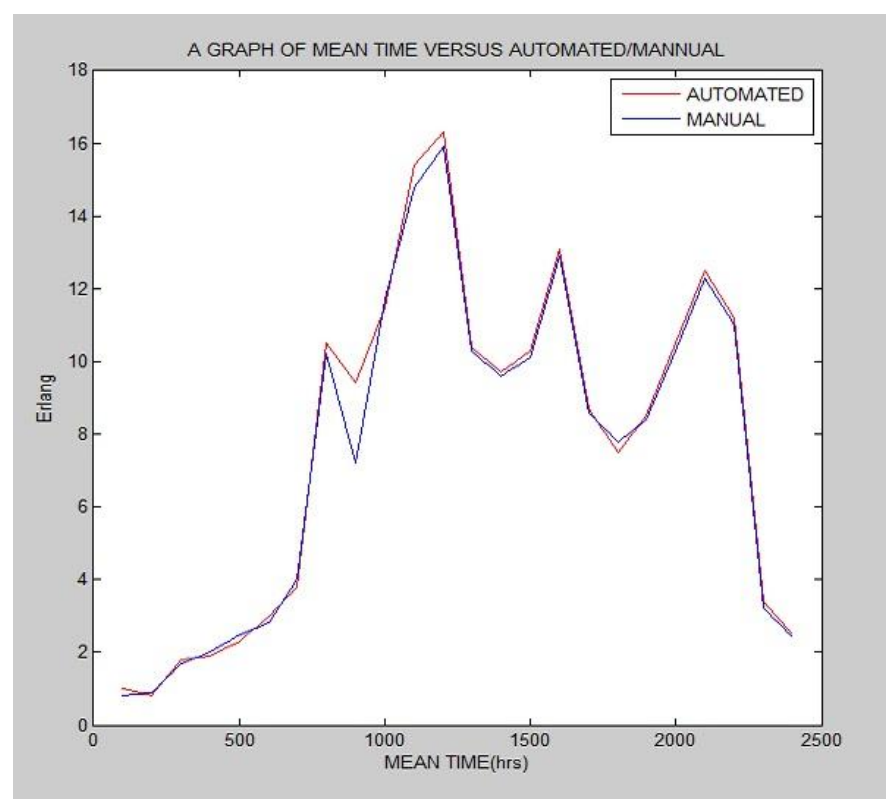

Figure 1: Comparative mean values of traffic carried (Erlang) using automated / manual devices in a day.

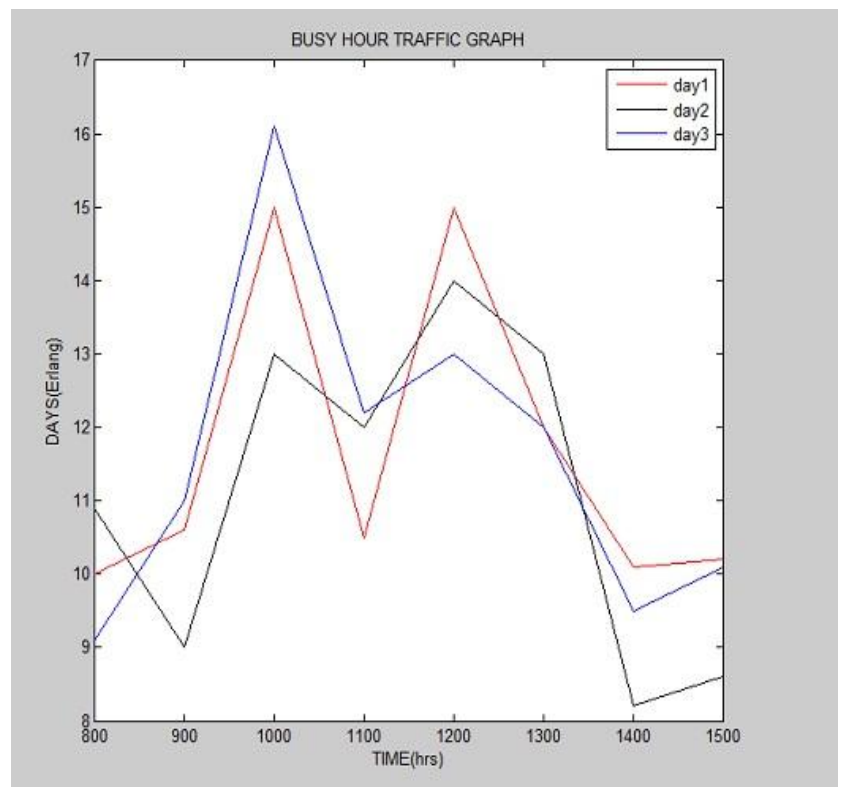

Figure 2: Mean traffic values in Erlang recorded from 0800 hours to 1500 hours for three consecutive days. 


\section{International Journal of Science and Research (IJSR) \\ ISSN (Online): 2319-7064}

Index Copernicus Value (2013): 6.14 | Impact Factor (2014): 5.611

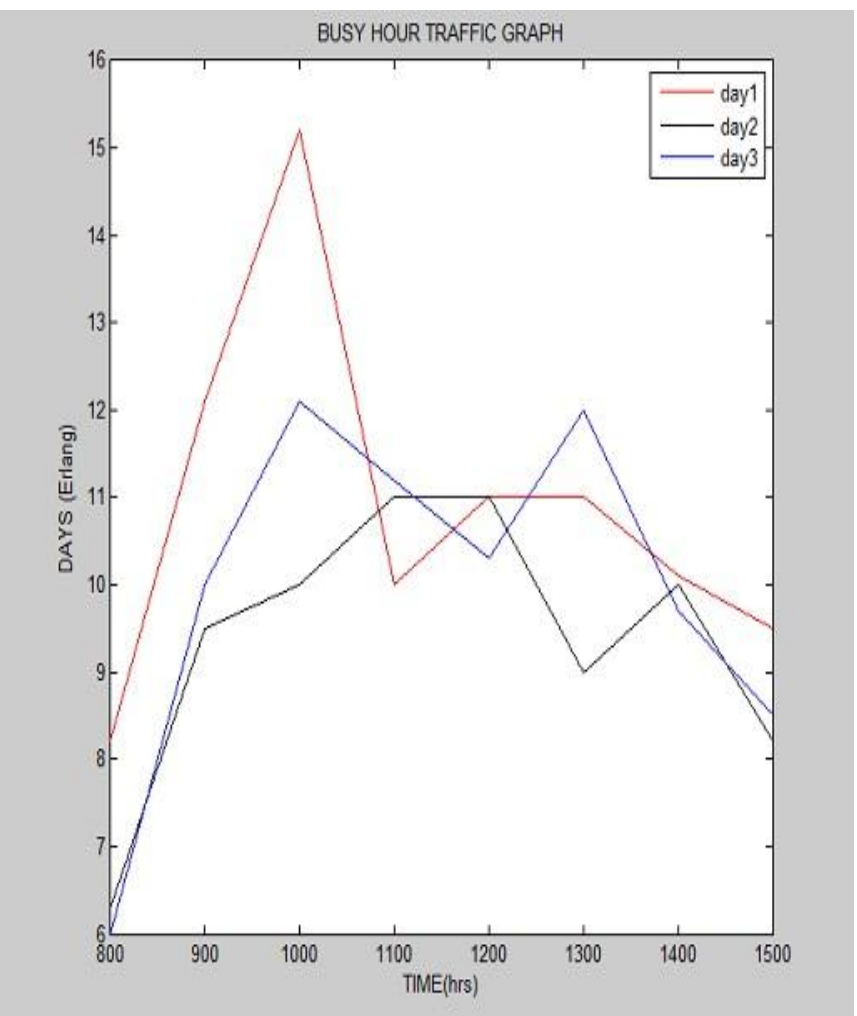

Figure 3: Mean traffic values in Erlang recorded from 0800 hours to 1500 hours for three consecutive days.

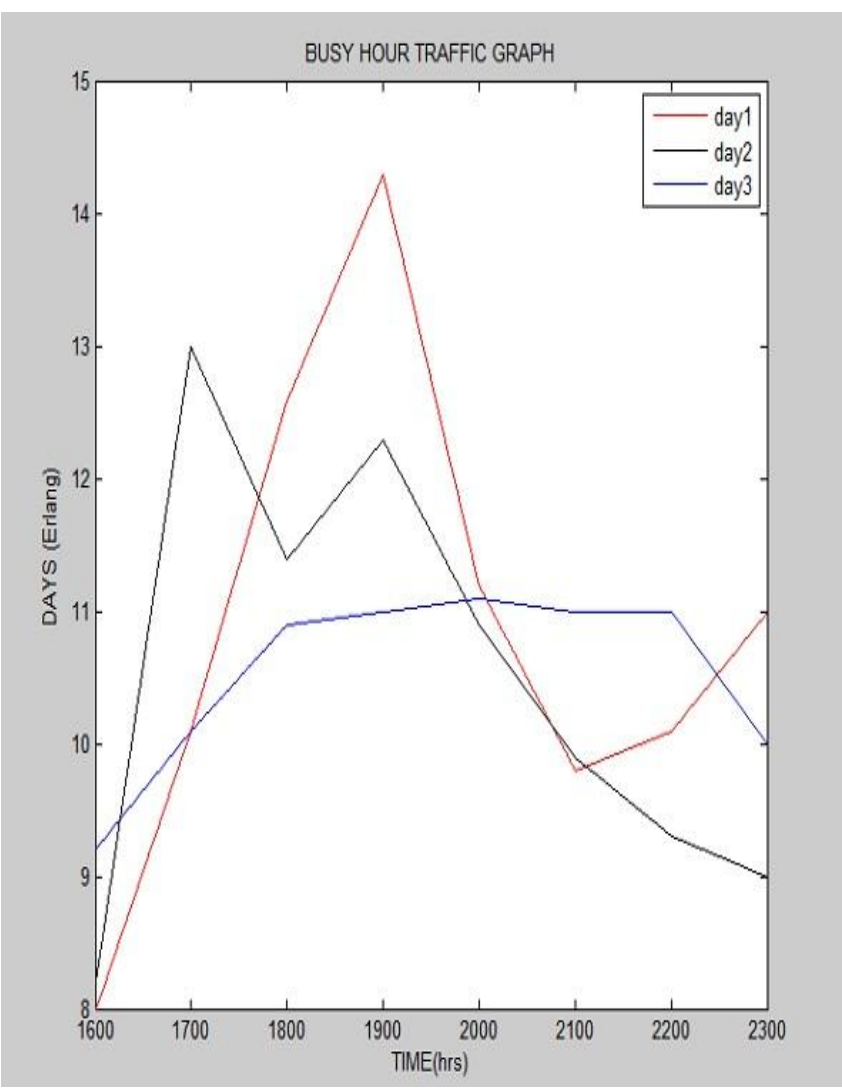

Figure 4: Mean traffic values in Erlang recorded from 1600 hours to 2400 hours for three consecutive days.

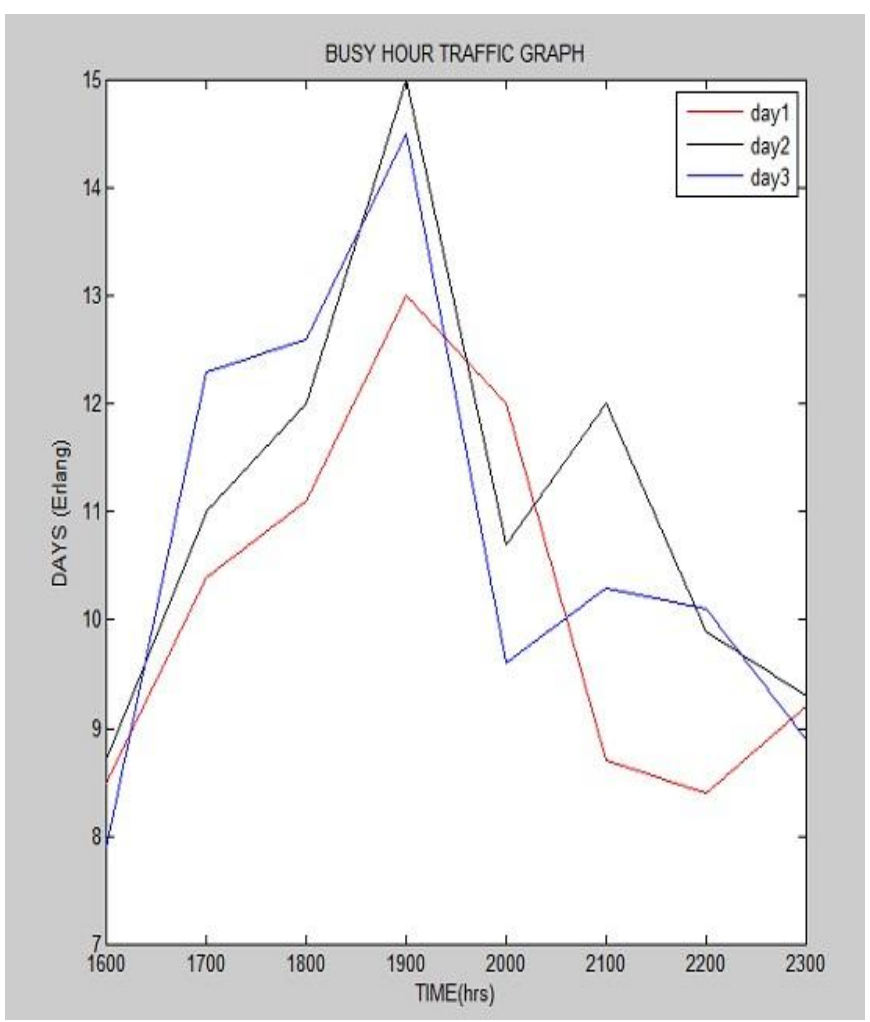

Figure 5: Mean traffic values in Erlang recorded from 1600 hours to 2400 hours for three consecutive days.

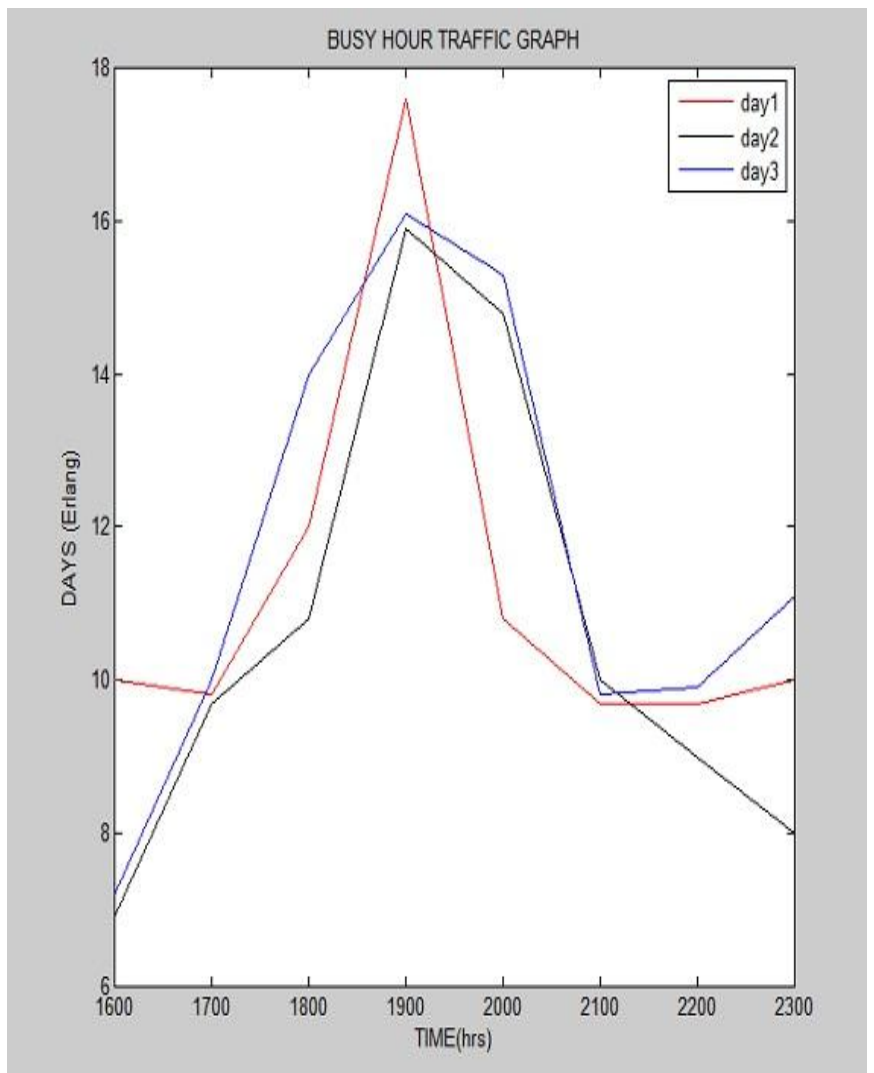

Figure 6: Mean traffic values in Erlang recorded from 1600 hours to 2400 hours for three consecutive days. 


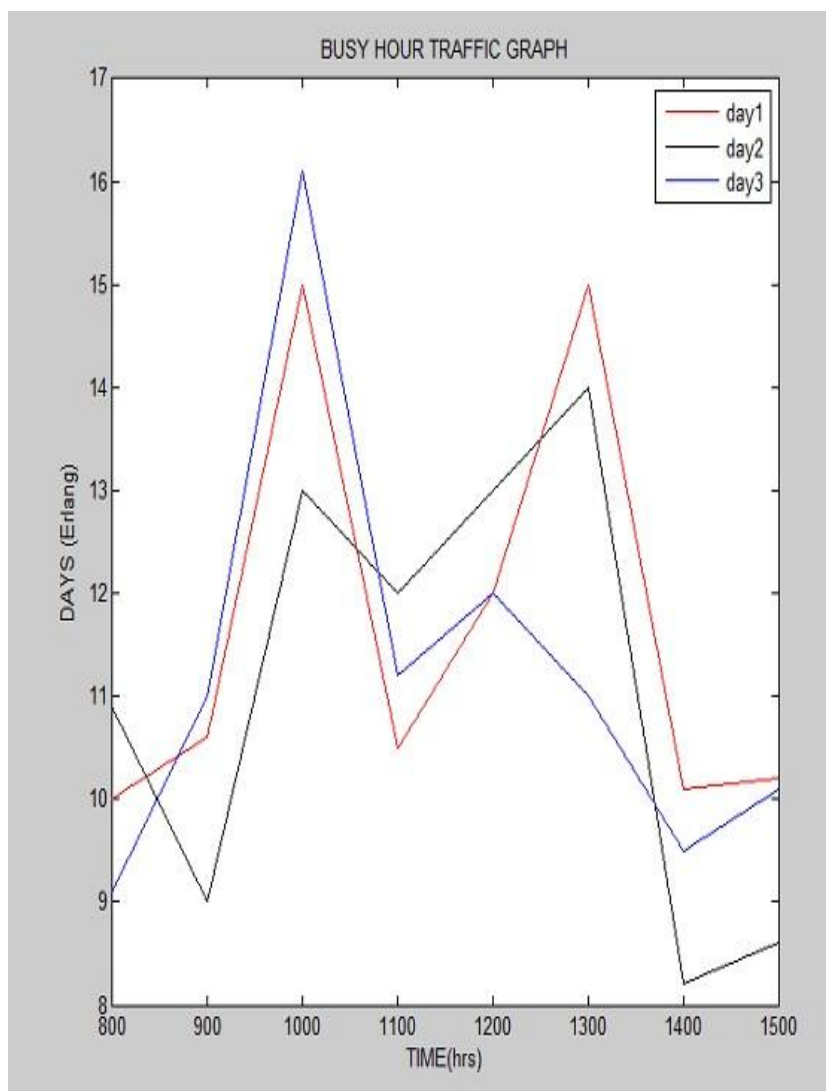

Figure 7: Mean traffic values in Erlang recorded from 0800 hours to 1500 hours for three consecutive days.

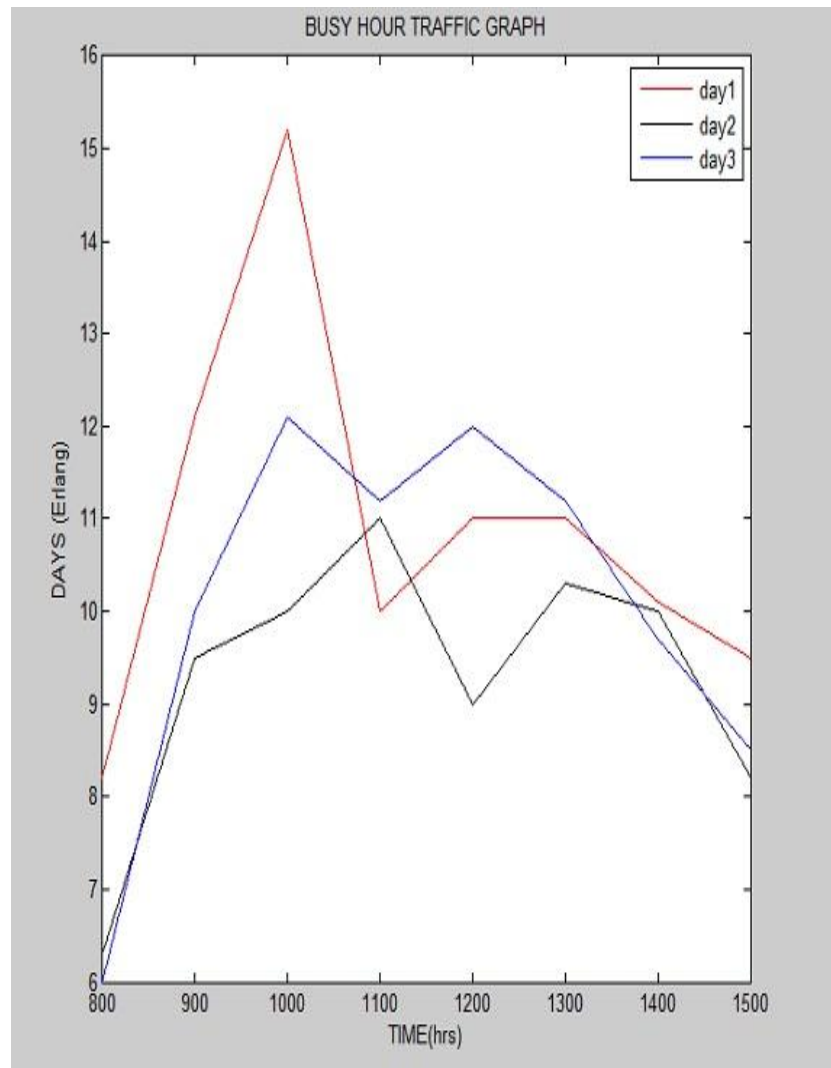

Figure 8: Mean traffic values in Erlang recorded from 0800 hours to 1500 hoursfor three consecutive days.

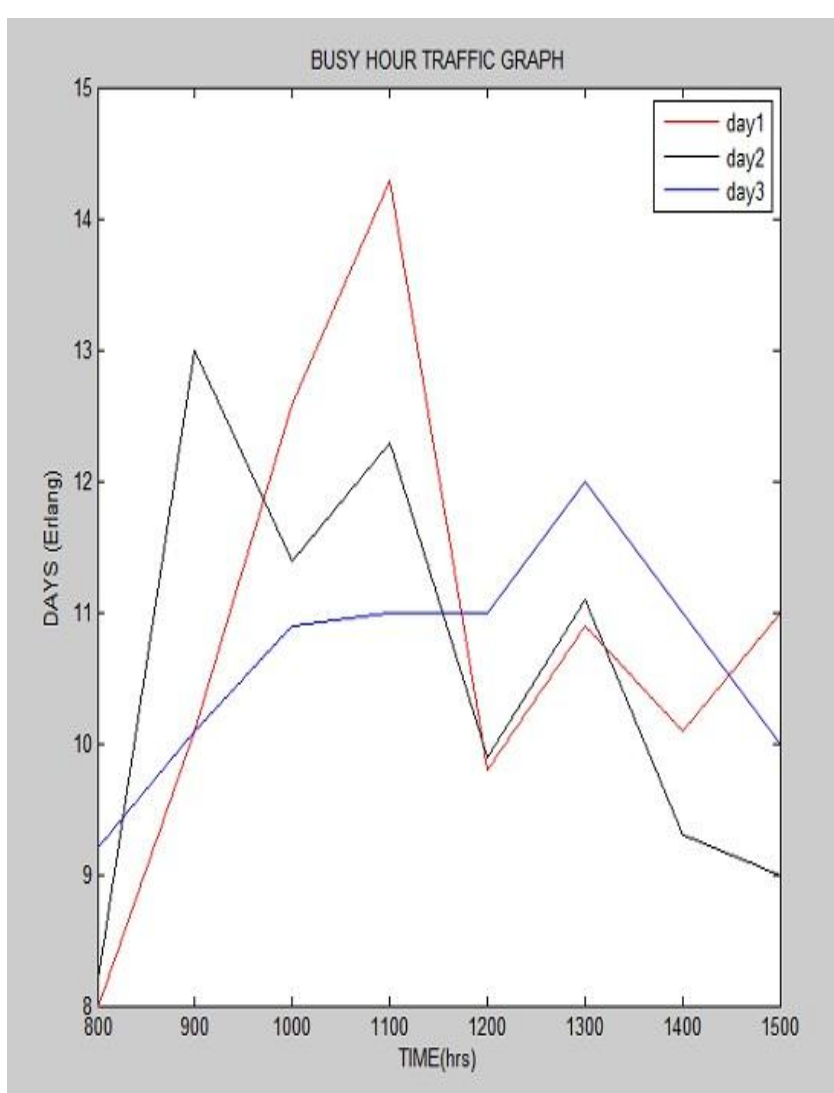

Figure 9: Mean traffic values in Erlang recorded from 0800 hours to 1500 hours for three consecutive days.

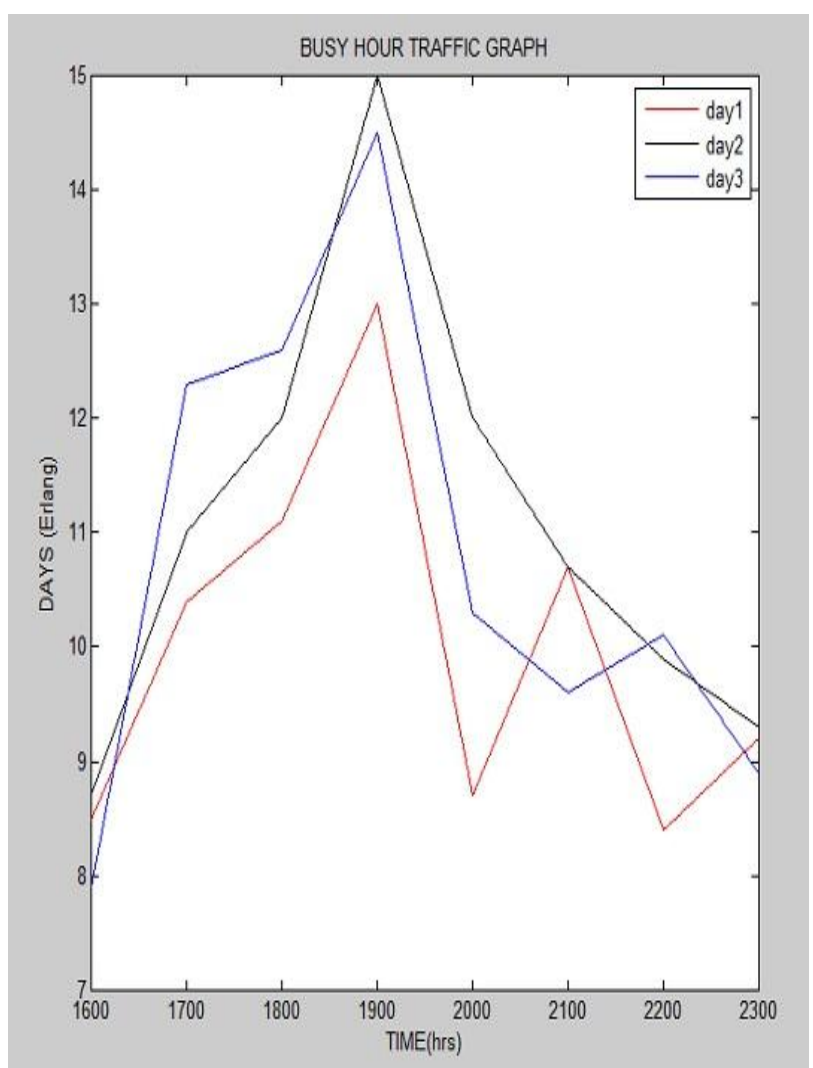

Figure 10: Mean traffic values in Erlang recorded from 1600 hours to 2400 hours for three consecutive days. 


\section{International Journal of Science and Research (IJSR) \\ ISSN (Online): 2319-7064}

Index Copernicus Value (2013): 6.14 | Impact Factor (2014): 5.611

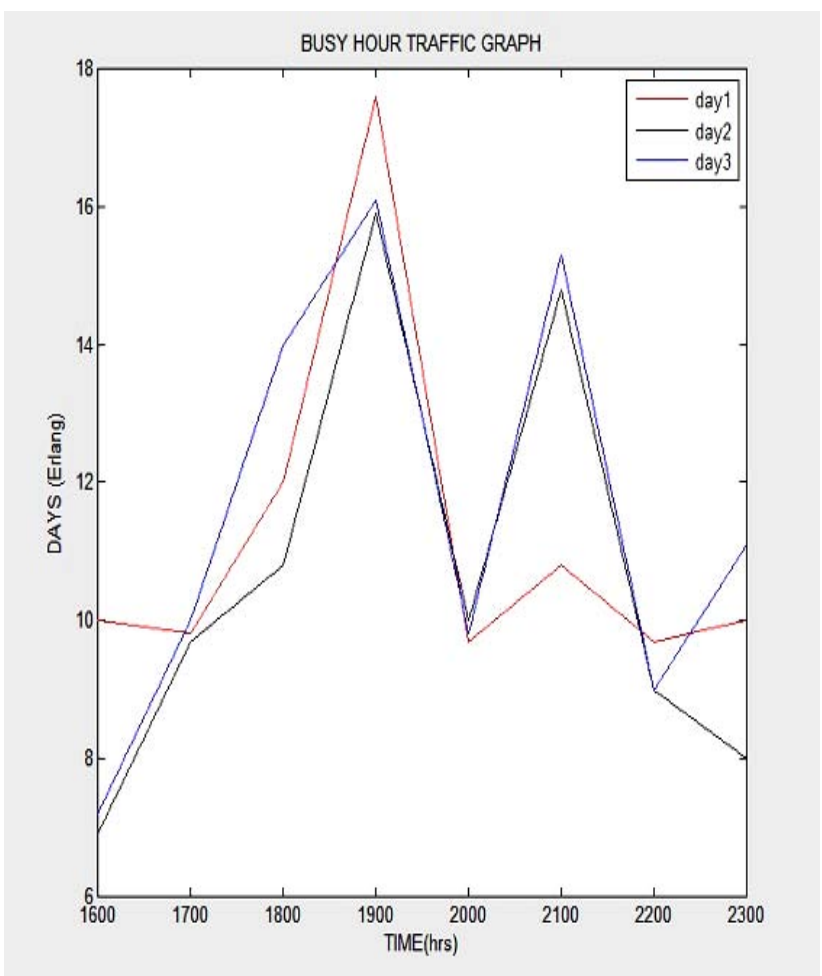

Figure 11: Mean traffic values in Erlang recorded from 1600 hours to 2400 hours for three consecutive days.

Fig. 1 is a comparative data acquired under similar conditions using two different measuring devices: computerized system and the manually operated device. Certain devices in common control switches such as markers and translators, though with very short holding times, are frequently seized. It is important to compare the accuracy of these manual devices with the automated values before deploying them. Measuring traffic data is expensive and earns no revenue for the network operator. It is therefore vital to organize it properly and efficiently in order to achieve the best GoS. As much as possible, measurements must be as accurate as possible, and any unavoidable inaccuracies or distortions must be clearly explained. In order to achieve both purposes, it is important to perform traffic measurements on a regular basis. Figures 2 to 11 are mean traffic values in Erlang, recorded using the time frame of the two major traffic busy period (dips) recorded in Figure 1. , 0800 hours to 1500 hours and 1500 hours to 2400 hours. The readings were taken in three consecutive days each under a stretch of six weeks. As shown in these figures, there were inconsistencies in the traffic occupancy of these days. They revealed unsuspected features not reflected by limited routing measurements that are usually dependent on the busy hour alone; or assist to pinpoint the area of trouble when congestion threatens. As demonstrated by Fig. 1 to Fig. 11, the traffic carried on each group or route is a direct measure of the flow of traffic in Erlang. The traffic carried (T) on each route is equal to the time-average of the number of busy circuits. If $\mathrm{E}$ is the grade of service offered and $\mathrm{A}$, the offered traffic, then: $\mathrm{T}=\mathrm{A}(1-\mathrm{E})$. When $\mathrm{E}$ is small, A approximates to $T$. Thus, if we can measure the average number of busy circuits, the traffic carried can be the estimate of the traffic offered.
The traffic curves are based on measurements using a medium - sized telephone exchange in a mixed business/residential area (60\% business) during working days and nights. The busiest hours were situated between 8 and $10.30 \mathrm{am}$. After a dip during the lunch period (from noon till $1 \mathrm{pm})$, traffic increased again but did not reach the morning level. There was also a peak at about 4pm. From these analyses, pure residential areas showed the highest peak occurring in the evening (between 6pm and 8pm). Private Internet users can shift the busy hour also to the evening period (7pm to $8.30 \mathrm{pm}$ ). Access to Internet can also increase the average call duration (up to 20 minutes to 2 hours) by a single subscriber. It is also possible that seasonal influences can occur. Example: traffic increases in touristic resorts during holidays.

Standard traffic theory proceeds on the assumption that during the busy-hour, traffic process is on statistical equilibrium with only random fluctuations and no systematic variations. That successive days in a measurement period are comparable. However, this study showed that this assumption cannot be true, considering the wide variability of traffic between busy hours and other period.

Furthermore, the study has demonstrated that if Fig. 2 to Fig. 11 were dependent on the busy hour traffic alone, necessary traffic details would have been omitted.

The time-consistent busy hour reduces the quantity of data to be collected and processed mostly in large capacity switching centers. This measure affects the overall result of data collected; which invariably distort the interpretation of data. However, in a mini switching module environment, this becomes less important, in view of the need to reduce data base and cost.

Busy - hour time consistent traffic scheme has worked in modern switching environment, using the simple assumption that the service provided during the determined busy hour was randomly distributed to other hours. Another assumption is that subscribers were more concerned with those times when they were significantly delayed or repeatedly blocked. Indications by this study showed that this scenario can be reliably practiced in a voice dependent bandwidth networks, and not in the present heterogeneous networks. It is therefore inferred that service criteria based on the average mean holding time, a consistent parameter used to determine the busy hour is a concept that is recommended for modification.

\section{Conclusion}

Often, customers are irritated whenever the network experiences peak load hours. Researchers have demonstrated that traffic peak loads follow the extreme value-distribution. However, new data system and technology have made measurements, collection and fitting of peak hour distributions to be easily processed with less dependence on the customized time/call consistent busy hour.

The EWSD-2000, switching system, used for this study routinely provides various reports without user intervention. 
Reports on each peak value observation were compared with control limits which are functions of the extreme engineering value distribution. Printed data were coded to identify observation on low and high day control limits. The rejection of a high day service usage by the switch is critical; and thus, the high day control limit of the switch counter was set at $\mathrm{P}$ (highest of $\mathrm{N}$ peaks $>\mathrm{x}$ ) $=1-\mathrm{P}^{\mathrm{N}}(\mathrm{x})<0.2$. The counter is incremented once for each value either exceeding the 14-day peak load threshold, $\bar{X}+1.86588$, or the 14-day peak usage capacity which is a function of the number of facilities provided and the service criteria. For any observation lower than these, the counter is decremented by one to a lower limit of zero. This standard is the universal engineering practice that is hereby considered obsolete and recommended for modified. The modification can form the basis for further studies on configuration of mega-traffic networks by stake holders.

From the above presentations, daily traffic analyses are deemed better in determining the actual network usage and real-time customers' behavior. These variables are very relevant for maintenance, operation and planning forecasts. The study, therefore, counters the current general practice of using fourth- nightly time consistent peak engineering value distribution.

\section{References}

[1] Abdulkadir, B. (2000). GSM: Why NITEL is interested. A House magazine Of Nigerian Telecommunications Limited, Nigeria. 28(3) - 19-21.

[2] Amaefule, E. (2006). Call Completion Rate: Nigerian Communications Commission threatens mobile operators, The Punch News Papers, Nigeria, p.19.

[3] Asouzu, C. (2004). Guide Lines for Trunk Traffic Management. Nigerian Communication Limited Operational Manual. Nigeria. 1. 22-24

[4] Bassey, D. E (2007): Teletraffic Handshake at Interconnection point and Traffic Refilling in Nigeria. NITEL Journal. A Quarterly Technical Publication, Vol. 62 14-18, 3.

[5] Bear, D. (1976). Telecommunications Traffic Engineering. London. Peter Perigrinus. 